\title{
MiR-195/-16 Family Enhances Radiotherapy via T Cell Activation in the Tumor Microenvironment by Blocking the PD-L1 Immune Checkpoint
}

\author{
Zhen Tao ${ }^{a}$ Shaohua Xu ${ }^{b}$ Hailong Ruanc Tao Wang ${ }^{d}$ Wen Song ${ }^{d}$ Li Qian $^{\text {e }}$ \\ Ke Chen ${ }^{c}$
}

\begin{abstract}
aDepartment of Radiation Oncology, Key Laboratory of Cancer Prevention and Therapy, Tianjin Medical University Cancer institute \& Hospital, National Clinical Research Center of Cancer, Tianjin, bepartment of Gynaecology, Shanghai First Maternity and Infant Hospital, Tongji University School of Medicine, Shanghai, 'Department of Urology, Union Hospital, Tongji Medical College, Huazhong University of Science and Technology, Wuhan, dDepartment of Urology, Tongji Hospital, Tongji Medical College, Huazhong University of Science and Technology, Wuhan, eInstitute of Translational Medicine, Medical College, Yangzhou University, Yangzhou, China
\end{abstract}

\section{Key Words}

Mir-195 • MiR-16 • PD-L1 • Tumor microenvironment • Immunotherapy • Radiotherapy

\begin{abstract}
Background/Aims: Radiotherapy is the standard treatment option for advanced prostate cancer. Unfortunately, despite significant advances in radiation delivery, prostate cancer radioresistance occurs in a large proportion of patients undergoing radiotherapy. As a way to enhance radiotherapy effectiveness, research advances into the mechanisms regulating the immune response have revived interest in combination radiation and immune-based therapies. Methods: miR-195/-16 family and PD-L1 levels were analyzed in samples from a GSE21032 data set. Kaplan-Meier analysis was used to evaluate the difference in biochemical recurrence-free survival associated with miR-195 and miR-16 expression. QRT-PCR and western blot were used to evaluate the miR-195, miR-16 and PD-L1 expression. Then, we used bioinformatics analysis and luciferase reporter assay to predict and confirm the miR-195 and miR-16 target gene. Finally, we elucidate the miR-195 and miR-16 function on immune evasion in the DU145/T cell co-culture model and syngeneic mouse model treated with radiotion through qRT-PCR, western blot, Flow cytometry and ELISA. Results: High levels of miR-195 and miR-16 were positively correlated with the biochemical recurrence-free survival of prostate cancer patients. miR-195 and miR-16 were inversely correlated with PD-L1, PD1, CD80 and CTLA-4 expression. Further mechanistic investigations revealed that miR-195

Z. Tao and S. Xu contributed equally to this work.

Ke Chen

and Zhen Tao

Department of Urology, Union Hospital, Tongji Medical College

Huazhong University of Science and Technology, Wuhan, Hubei (China)

E-Mail shenke@hust.edu.cn, ztao@tmu.edu.cn
\end{abstract}


and miR-16 inhibited PD-L1 expression. Additionally, restoration of miR-195 and miR-16 expression enhanced radiotherapy via $T$ cell activation in the tumor microenvironment by blocking PD-L1 expression. This synergistic effect of immunotherapy and radiotherapy was associated with the proliferation of functional cytotoxic CD8 + T cells and inhibition of myeloidderived suppressor cells and regulatory T cells. Conclusions: Our data revealbiological and functional interactions between immunotherapy and radiotherapy through the miR-195/-16 family regulatory cascade.

(C) 2018 The Author(s)

Published by S. Karger AG, Basel

\section{Introduction}

Radiotherapy is the standard treatment option for both organ-confined and regionally advanced prostate cancer. Unfortunately, despite significant advances in radiation delivery procedures, prostate cancer radioresistance occurs in a large number of patients undergoing radiotherapy [1]. As a way to enhance radiotherapy effectiveness, research advancesinto the mechanisms regulating the immune response have revived interest in combination radiation and immune-based therapies.

The immune response affects cancer development by acting as an extrinsic tumor suppressor that restrains tumor expansion or destroys tumors [2]. However, tumor cells can escape immune surveillance. T-lymphocyte associated antigen-4 (CTLA-4) and programmed death-1 (PD-1) are the two immunoregulatory proteins expressed on T cell membranes [3-4]. CD80, the CTLA-4 receptor ligand, is expressed in dendritic cells, whereas PD-1 ligand 1 (PDL1), the PD-1 ligand, is expressed in tumor and/or macrophage cells [5]. The interactions of CD80 with CTLA-4 and of PD-L1 with PD-1 inhibit CD8+ cytotoxic T lymphocyte survival and proliferation and affect the function of tumor-infiltrating $\mathrm{T}$ cells, ultimately suppressing the immune response and inducing immune tolerance of cancer [6]. Radiotherapy exerts some potentially anti-therapeutic effects by inducing tumor cell that express PD-L1, secrete TGF- $\beta$, and induce regulatory $\mathrm{T}$ cells (Tregs) [7]. These findings suggest a potential link between radiotherapy and immunoresistance in prostate cancer.

miR-195 and miR-16 are members of the miR-15/-16/-195/-424/-497/-503 family. miR-195 and miR-16 overexpression increases radiosensitivity of cancer cell [8-9]. miR195 inhibits human prostate cancer progression by targeting RPS6KB1, and miR-16 acts as a tumor suppressor gene in prostate cancer by controlling cell survival, proliferation and invasion via BCL2, CCND1 and WNT3A [10-11]. However, it is unclear whether the miR-195/-16 family is involved in the regulation of the immune response. In this study, we demonstrated both in vitro and in vivo that restoration of miR-195 and miR-16 expression enhanced radiotherapy via $\mathrm{T}$ cell activation in the tumor microenvironment by blocking the PD-L1 immune checkpoint.

\section{Materials and Methods}

Cell culture, Plasmid construction, Reporter genes, Reagents, Expression vectors, and DNA transfection

The prostate cancer cell lines PC-3, DU145 and Trampc1 were purchased from The Cell Bank of Chinese Academy of Science. Cells were maintained in a medium of RPMI 1640 supplemented $10 \%$ FBS and $1 \%$ penicillin/streptomycin and were kept in $5 \% \mathrm{CO} 2$ incubator at $37^{\circ} \mathrm{C}$. PLe-miR-SCR lentivirus vector and pLe-miR-195 and pLe-miR-16 were purchased from Open Biosystems, AL, USA. Cells infected with pLe-miR-195 or pLe-miR-16. Has-miR-195 and has-miR-16 mimics were purchased from Guangzh Ribobio Co., LTD, China. Hsa-miR-195, hsa-miR-16 mincis and hsa-miR-SC were transfected into cells using Lipofectamine RNAiMAX (Invitrogen, CA, USA) according to the manufacturer's instructions. Protein lysates and total RNAs were collected $48 \mathrm{~h}$ after the transfection. The expression levels of miRNAs were verified by stem-loop qRT-PCR analysis. All cell lines were tested and free of mycoplasma contamination (MycoAlert Mycoplasma Detection Kit, Lonza).

\section{KARGER}




\section{Cellular Physiology Cell Physiol Biochem 2018;48:801-814 \begin{tabular}{l|l} 
and Biochemistry Published online: July 20, 2018 & $\begin{array}{l}\text { (c) } 2018 \text { The Author(s). Published by S. Karger AG, Basel } \\
\text { www.karger.com/cpb }\end{array}$
\end{tabular}}

Tao et al.: Mir-195/-16 Family Modulated Immune Response

Blood DCs isolation

Blood DCs were isolated from healthy human donors using a Human Blood Dendritic Cell Isolation Kit II (Miltenyi Biotec, No. 130-091-379) following the manufacturer's instruction.

\section{Tissue samples}

A collection of 40 different kinds of fresh-frozen prostate cancer tumor specimens and 20 tumor normal adjacent tissues were from Tongji Hospital, Tongji Medical College, Huazhong University of Science and Technology. All tumor samples were collected immediately after the surgical removal and snap-frozen in liquid nitrogen [12].

\section{Biochemical recurrence-free survival analysis in GSE21034 data base.}

A normalized mRNA expression dataset for Prostate Adenocarcinoma was downloaded from the cBioPortal for cancer genomics and used to evaluate expression of miR-195/16 family and PD-L1 transcript levels [13]. This dataset includes mRNA and miRNA profiles for 131 primary prostate cancer patient samples. Biochemical recurrence-free survival analysis was calculated for these transcripts for primary tumor samples.

\section{Western Blot Analysis.}

Cells were lysed in RIPA buffer with protein inhibitors. Total protein (20-40g) from each sample was electrophoresised on $8 \%$ SDS-PAGE gel and transferred to a nitrocellulose membrane. The membranes were blocked in 5\% nonfat milk and probed with primary antibodies overnight at $4{ }^{\circ} \mathrm{C}$. The membranes were washed and probed with secondary antibody conjugated to horseradish peroxidase and the developed with enhanced chemiluminescence (Thermo Scientific).

\section{Quantitative real-time PCR}

Total RNA was isolated using Trizol Reagent (Life Technologies, Carlsbad, CA, USA) according to the manufacturer's instructions. cDNA were synthesized with TaqMan Reverse Transcription Reagents (Life Technologies). The expression levels of miRNAs were analyzed using Taqman MicroRNA Assay Kits (Applied Biosystems, Foster City, CA) specific for hsa-miR-195, has-miR-16 and PD-L1 mRNAs. The fold stimulation was determined using the comparative cycle threshold method (method ( $\left.2^{- \text {ब回T }}\right)$ ). All experiments were performed in triplicate [14].

\section{Luciferase reporter assay}

Luciferase reporter assay was done as described previously [15]. PD-L1 3'-UTR regions that contained predicted miR-195 and miR-16 binding sites were amplified by PCR from genomic DNA, and the PCR fragments were inserted into untranslated region (UTR) downstream of the luciferase gene in the pMIRreporter luciferase vector (Ambion). Luciferase reporter plasmid, $\beta$-galactosidase ( $\beta$-gal) plasmid, and hsamiR-195, hsa-miR-16 or negative control precursor were cotransfected into cells using Lipofectamine 2000 (Invitrogen). Luciferase activity was measured 48 hours after transfection using $\beta$-gal for normalization.

\section{Preparation of DU145 cell lysate}

DU145 cell lysate was done as described [16]. Confluent cultures of DU145 cell line was incubated with $0.01 \%$ EDTA-solution for $10 \mathrm{~min}$, carefully detached with a cell scraper, washed twice in PBS, and resuspended at a densiry of $5 \times 10^{6} / \mathrm{ml}$ in serum-free mediun. The cell susupensions were frozen at $-80^{\circ} \mathrm{C}$ and disrupted by four freeze-thaw cycles. For the removal of crude debirs, the lysate was centrifuged for 10 min at $300 \times \mathrm{g}$. The supernatant was collected and passed through a $0.2-\mathrm{mm}$ filter.

\section{DU145/T co-culture model}

DU145/T cells co-culture model was done as described previously [17]. Peripheral blood mononuclear cells (PBMCs) from healthy human donors were isolated using Lymphoprep density gradient centrifugation (Accurate Chemical). Human PBMCs were plated at a density of $2 \times 10^{6}$ well $^{-1}$ in 6 -well plates and stimulated with DU145 cell lysate, anti-CD3e $\left(10 \mu \mathrm{g} \mathrm{mL}^{-1}\right)$ and anti-CD28 $\left(2 \mu \mathrm{g} \mathrm{mL}^{-1}\right)$ for $48 \mathrm{~h}$ to promote $\mathrm{T}$ cell activation. The T cell activation protocol was provided by eBioscience (http://www.ebioscience.com/cell-type/t-cells. htm). DU145 cells that stably expressed miR-195 and miR-16. Stimulated human PBMCs were subsequently 


\section{Cellular Physiology Cell Physiol Biochem 2018;48:801-814 \begin{tabular}{l|l|l} 
and Biochem 10.1159/000491909 & $\begin{array}{l}\text { C } 2018 \text { The Author(s). Published by S. Karger AG, Basel } \\
\text { www.karger.com/cpb }\end{array}$
\end{tabular} \\ Tao et al.: Mir-195/-16 Family Modulated Immune Response}

harvested and purified by Lymphoprep density gradient centrifugation, and co-cultured with the DU145 cells at a 10:1 ratio for $16 \mathrm{~h}$. The DU145 cells were then sorted by FCM. The relative expression levels of PDL1 in the DU145 cells were determined by qRT-PCR assay. Co-culture media were assayed for TNF- $\alpha$, IFN- $\gamma$, IL-2, IL-10, IL-1 $\beta$ and TGF- $\beta$ using a cytokine ELISA assay.

\section{T cell apoptosis assay}

PBMCs were isolated from healthy human donors using Lymphoprep density gradient centrifugation (Accurate Chemical). PBMCs were plated at a density of $2 \times 10^{6}$ well $^{-1}$ in 6 -well plates and stimulated with DU145 cell lysate, anti-CD3e $\left(10 \mu \mathrm{g} \mathrm{mL} \mathrm{mL}{ }^{-1}\right)$ and anti-CD28 $\left(2 \mu \mathrm{g} \mathrm{mL} \mathrm{m}^{-1}\right)$ for $48 \mathrm{~h}$ to promote $\mathrm{T}$ cell activation. The DU145 cells were first exposed to radiation for $48 \mathrm{~h}$. Stimulated PBMCs were subsequently harvested and purified by Lymphoprep density gradient centrifugation, and then co-cultured with DU145 cells at a 10:1 ratio for $16 \mathrm{~h}$. Ten micrograms per milliliter anti-PD-L1 were added to the indicated wells to examine PD-1-specific CD8+ T cell apoptosis. The PBMCs were subsequently harvested and stained with PEconjugated PD-1, Alexa Fluor 488-conjugated annexin V and APC-conjugated CD8 antibodies, respectively. PD-1-dependent CD8+ T cell apoptosis was calculated as the percentage of annexin V+ cells in the gated PD-1+/CD8+ population [17].

Enzyme-linked immunosorbent assays (ELISA)

The production of T cell cytokine was detected by cytokine ELISA using the human or mouse ELISA kit (ExCell Bio, Shanghai, China) as described [17].

Syngeneic orthotopic prostate cancer model

Syngeneic orthotopic prostate cancer model was done as described previously [17]. Trampc1 cells were culture, harvested, and suspended in PBS. For flank injections, a total of $0.5 \mathrm{ml}$ containing $5 \times 10^{6}$ cells was injected subcutaneously into the flank of 6- to 8-week-old Tramp mice (Chinese Academy of Sciences at Beijin, China). Tumors were allowed to develop over for 8 days, until tumor diameter was approximately $0.5 \mathrm{~cm}$. On day 9 , the mice were treated with 20 Gy radiation using a PanTak $310 \mathrm{keV}$ X-ray machine at 0.25 $\mathrm{mm} \mathrm{Cu}$ plus $1-\mathrm{mm} \mathrm{Al}$ added filtration at $125 \mathrm{cGy} / \mathrm{min}$. The mice serum was collected at $72 \mathrm{~h}$ after radiation treatment. Tumor dimensions were measured every 3 days. Analyses included assessment of tumor growth and survival. Tumors tissues were used for enumeration and characterization of MDSCs, Tregs, and CD8+ T cells memory subsets. Mice serum was assayed for IL-10, TNF- $\alpha$, IFN- $\gamma$, and TGF- $\beta$ by cytokine ELISA assay.

\section{Statistical analysis.}

All statistical analyses were performed using EXCEL 2010 (Microsoft, USA). The results reported as mean \pm SD. Statistical analyses were conducted using Student's $t$-test and Pearson Correlation Coefficient. $p$ $<0.05$ were considered statistically significant.

\section{Results}

miR-195 and miR-16 expression are inversely correlated with PD-L1 levels in prostate cancer

miRNAs act as post-transcriptional modulators of gene expression mainly by binding to the 3' untranslated region (3'-UTR) of their target genes and have been shown to play important roles in regulating the immune response [18]. Through the public database microRNA.org, we first analyzed the 3'-UTR sequences of PD-L1, PD-1, CD80 and CTLA-4, finding that PD-L1, which is an immune checkpoint gene, is potentially targeted by miR$15 a /-15 b /-16 /-195 /-424 /-497 /-503$ family. To evaluate the physiological interactions between the miR-15a/-15b/-16/-195/-424/-497/-503 family and PD-L1/PD-1/CD80/ CTLA-4 expression, we performed in silico analysis using a GSE21032 dataset composed of 131 primary prostate cancer patient samples. We also conducted Kaplan-Meier analysis by separating patients into two groups (higher than the 50th percentile and lower than the 50th percentile) according to their miRNA expression levels, to determine whether biochemical recurrence(BCR)-free survival was correlated with the level of miR-15a/$15 b /-16 /-195 /-424 /-497 /-503$ family members in tumors. The results indicated that 
Fig. 1. miR-195 and miR-16 are inversely correlated with PD-L1 levels in prostate cancer patients. (A) Heat map depicting samples from GSE21034 combined human prostate cancer microarray datasets that were assigned to prostate cancer gene expression subtypes $(\mathrm{n}=150)$. Kaplan-Meier analysis indicating that patients with samples with highly expressed (> $50 \%$ ) miR-195 or miR-16 had significantly better BCR-free survival. (B) miR-195 and miR-16 levels are inversely correlated with PD-L1, PD-1, CD80 and CTLA4 expression levels in human prostate cancer. (C) PD-L1 and PD-1 expression levels are positively correlated with CD80 and CTLA-4 expression levels in human prostate cancer. (D) qRT-PCR was performed to determined miR-195, miR-16 and PD-L1 expression in 20 normal prostate tissues and 40 primary prostate tumors. (E) qRT-PCR was performed to determine miR-195, miR-16 and PD-L1 expression in 40 primary prostate tumors. Spearman's rank correlation analysis identified an inverse correlation between miR195/-16 and PD-L1 levels in the prostate cancer tumor samples.

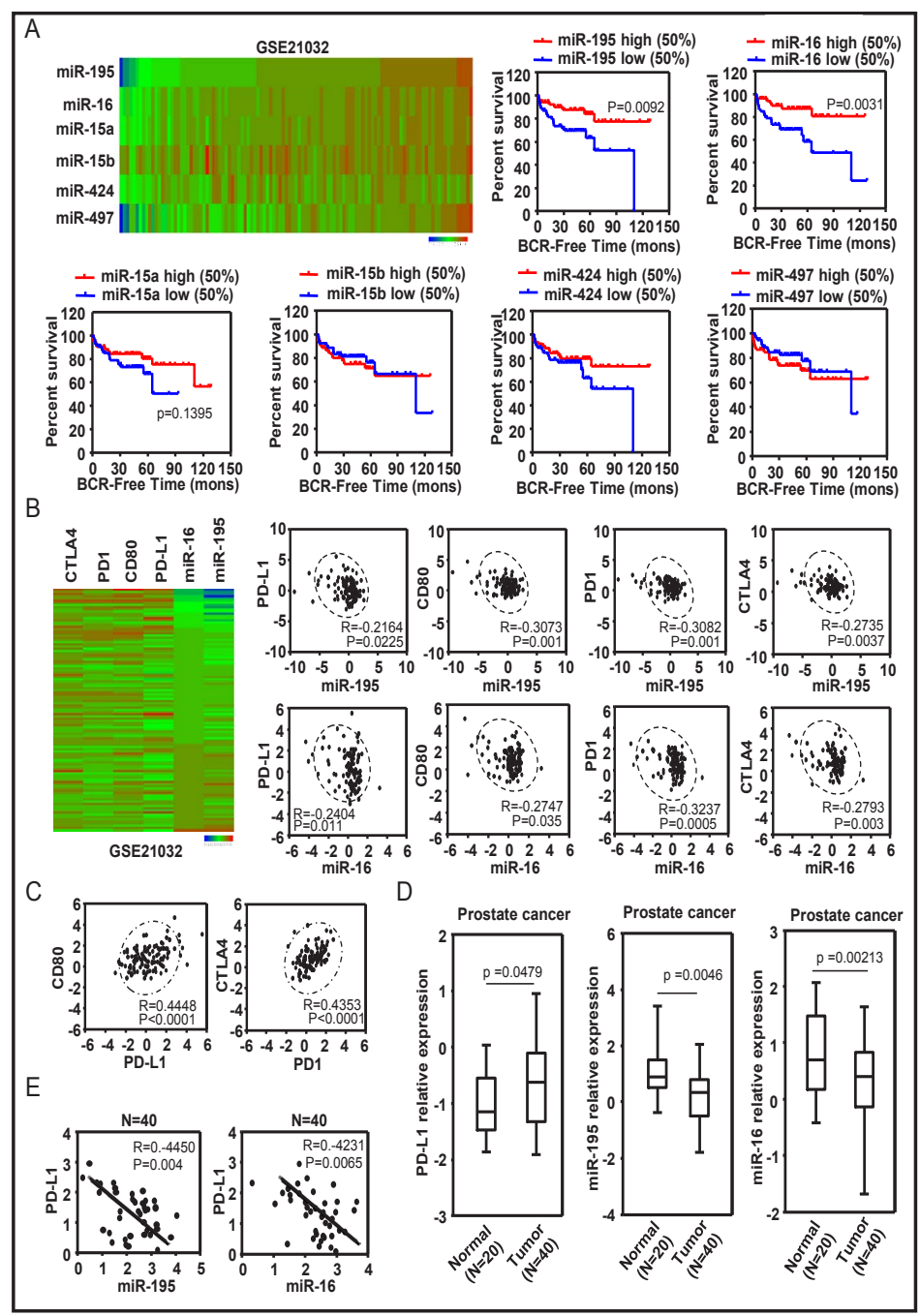

patients with tumors that expressing high levels of miR-195 and miR-16 had a longer BCRfree survival (Fig. 1A). In addition, miR-195 and miR-16 were negatively associated with PDL1, PD-1, CD80 and CTLA-4 expression (Fig. 1B), whereas a positive correlation was found among the expressions of PD-L1, PD-1, CD80 and CTLA-4 (Fig. 1C).

To further confirm the interaction between miR-195/-16 and PD-L1, we examined miR-195, miR-16 and PD-L1 levels with quantitative real-time polymerase chain reaction (qRT-PCR) in prostate cancer samples. As shown in Fig. 1D, the miR-195/-16 levels were significantly decreased and the PD-L1 levels were dramatically increased in primary prostate tumors compared with normal tissues. The miR-195 and miR-16 levels were inversely correlated with PD-L1 (Fig. 1E). Taken together, these findings suggested that miR-195 and miR-16 modulated the immune response by interacting with the PD-L1/PD-1 immune checkpoint in prostate cancer.

\section{$P D-L 1$ is regulated by miR-195 and miR-16}

To validate the bioinformatics analysis, which indicated that PD-L1 was a potential target of miR-195 and miR-16, we performed luciferase reporter assays with human wildtype PD-L1 3'-UTR and mutants with deletions of the putative miR-195 and miR-16 binding sequences. As shown in Fig. 2A-B, overexpression of miR-195 and miR-16 inhibited wild-type but not mutant luciferase reporter activity in DU145 and PC-3 cells, which suggested that miR-195 and miR-16 targeted PD-L1 through the 3'-UTR binding site. Western blot analyses 
Fig. 2. PD-L1 is directly regulated by miR-195 and miR-16. (A-B) miR-195 and miR-16 targeting sites in the 3-'UTR of human PD-L1 are shown. Luciferase vectors containING the human wild-type (WT) and mutant (MUT) PD-L1 3'- UTR regions were co-transfected into DU145 cells with miR-195 or miR-16. Relative Luciferase/Renila activities were analyzed in the cells $48 \mathrm{~h}$ after the transfection. (C) Du145 and PC3 cells were transfected with miR-195 or miR-16. miR-195 and miR-16 mRNA levels were determined via qRT-PCR assay. The expression levels of PD-L1 were determined by western blotting. Results represent the mean \pm standard error of the mean (SEM) from three independent experiments. ${ }^{*} \mathrm{p} \leq 0.05$, ** $\mathrm{p} \leq 0.01$.

subsequently demonstrated that miR-195 or miR-16 overexpression in DU145 and PC-3 human cancer cells decreased the protein levels of PD-L1 (Fig. 2C). Taken together, these findings indicated that miR-195 and miR-16 suppressed PD-L1 expression.

miR-195 and miR-16 regulates cytokine secretion in the tumor microenvironment through a PD-L1-dependent pathway
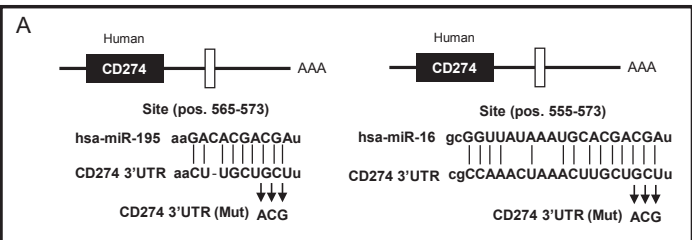

B
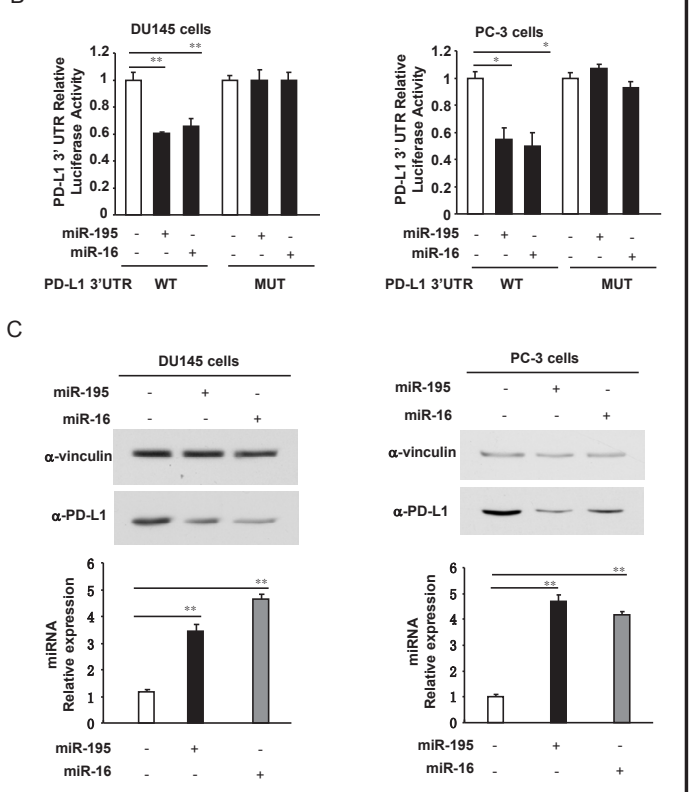

The release of cytokines in the tumor

microenvironment is critical in many aspects of T cell function [19-20]. The PD-L1/PD-1 immune checkpoint pathway inhibits the anti-tumor immune response of $\mathrm{T}$ cells [6], and PD-L1 preferentially modulates the secretions of regulatory cytokines in the tumor microenvironment [21]. Therefore, we tested whether an increased PD-L1 level through miR-195 and miR-16 signaling in prostate cancer affected the tumor microenvironment. We established a DU145/T cell co-culture model. Co-culture T cells with DU145 cells stably expressing miR-195 and miR-16 decreased IL-1 $\beta$, IL-10 and TGF- $\beta$ secretion and increased TNF- $\alpha$, IFN- $\gamma$ and IL-2 in the culture media, whereas PD-L1 depletion through a PD-L1blocking antibody (anti-PD-L1) abolished miR-195- and miR-16-mediated cytokine secretion (Fig. 3A-F). These findings suggested that miR-195 and miR-16 modulated cytokine secretion in the tumor microenvironment by blocking a PD-L1/PD-1-dependent pathway.

miR-195 and miR-16 influences PD-L1-associated T cell apoptosis in the DU145/T cell coculture model

Previous studies demonstrated that the expression of PD-L1 was directly repressed by miR-195 and miR-16. To investigate the combined effect of miR-195/-16 and radiation in human prostate cancer, we established DU145 cells stably expressing miR-195 and miR-16, and then treated them with radiation or anti-PD-L1. Flow cytometer (FCM) analysis showed that cell surface PD-L1 expression was significantly higher in radiation treated DU145 cells (48h after 20Gy radiation) compared with parental cells (Fig. 4A). Restoration of miR195 and miR-16 expression in radiation treated DU145 cells decreased cell surface PD-L1 expression, whereas anti-PD-L1 treatment abolished miR-195 and miR-16 overexpressionmediated PD-L1 repression in the radiation treatment group (Fig. 4A).

It has been demonstrated that the PD-L1/PD-1 pathway inhibits the anti-tumor immune response of $\mathrm{T}$ cell. To determine whether miR-195 and miR-16 affect the $\mathrm{T}$ cell response by regulating PD-L1 expression, we performed DU145/T cell co-culture experiments. Co-culture of T cells with radiated DU145 cells increased PD-1+/CD8+ T cell apoptosis 
Fig. 3. miR-195 and miR-16 regulate cytokine secretion in the tumor microenvironment through a PD-L1-dependent pathway. (A-F) DU145 cells with stable overexpression of miR-195 or miR-16 treated with anti-PD-L1. After $24 \mathrm{~h}$, T cells were then co-cultured with DU145 cells for $24 \mathrm{~h}$. Co-culture medium was assayed for TNF- $\alpha$, IFN- $\gamma$, IL-2, IL-10, IL-1 $\beta$ and TGF- $\beta$ by cytokine enzyme-linked immunosorbent assay (ELISA). Results represent the mean \pm SEM from three independent experiments. $* * \mathrm{p} \leq 0.01$.

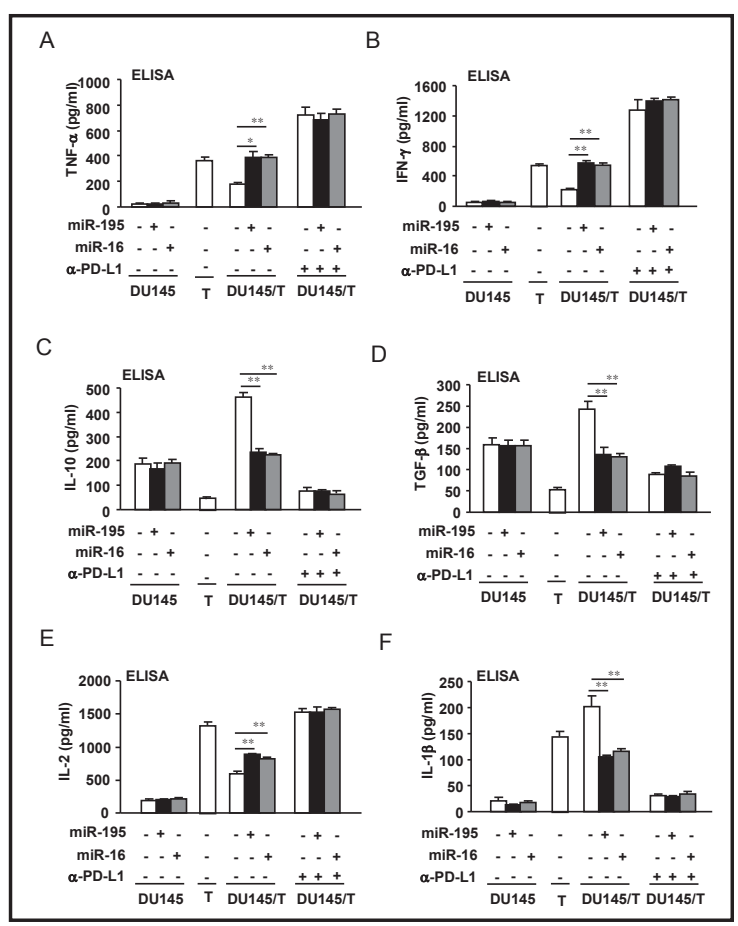

Fig. 4. miR-195 and miR-16 influences PD-L1associated $\mathrm{T}$ cell apoptosis in DU145/T cell coculture model. (A) DU145 cells with stable overexpression of miR-195 and miR-16 were first exposed to radiation for $48 \mathrm{~h}$ in the presence or absence of anti-PD-L1. The expression of cell surface PD-L1 was determined by FCM in DU145 cells. Results represent the mean \pm SEM from three independent experiments. ${ }^{*} \mathrm{p} \leq 0.05,{ }^{* *} \mathrm{p} \leq 0.01$. (B) $\mathrm{T}$ cells were subsequently co-cultured with DU145 cells for $24 \mathrm{~h}$. T cells were sorted by FCM, stained with PE anti-PD-1, Alexa Fluor 488 anti-annexin V and APC anti-CD8, and analyzed by flow cytometry for T cell apoptosis in the PD-1+/CD8+ population. ${ }^{* *} \mathrm{p} \leq 0.01$. Results represent the mean \pm SEM from three independent experiments, and the densitometric level of the apoptosis ratio is shown.

compared with $\mathrm{T}$ cells cultured with intact DU145 cells (Fig. 4B). However, in DU145 cells overexpressing miR-195 and miR-16, the apoptosis of PD-1+/CD8+ T cells induced by radiation treatment was completely abolished (Fig. 4B). Similar

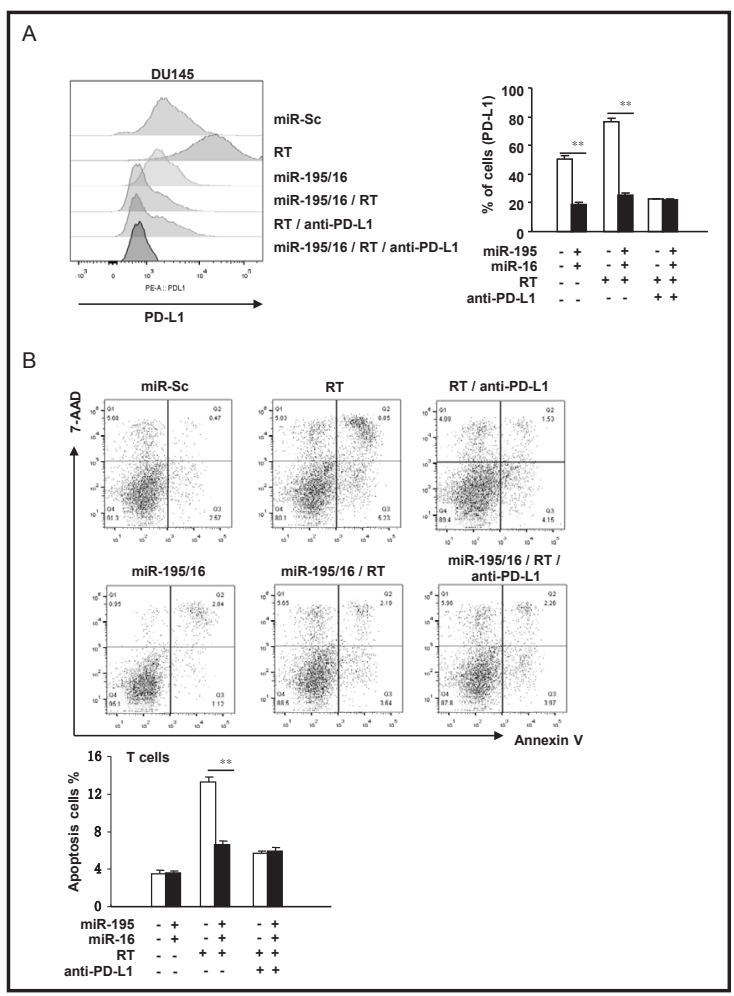
to anti-PD-L1, miR-195 and miR-16 were also able to mediate apoptosis in PD-1+/CD8+ T cells, which suggested that they decreased radiation-induced $\mathrm{PD}-1+/ \mathrm{CD} 8+\mathrm{T}$ cell apoptosis by blocking PD-L1. These results also showed that radiotherapy-induced immune-resistance could be reversed by miR-195 and miR-16 overexpression. These findings indicated that miR-195 and miR-16 improved the therapeutic efficacy of radiation-resistance by suppressing PD-L1. 
PD-L1 is required for the efficacy of miR-195 and miR-16 treatment in Trampc1 tumors

Next, we analyzed the mouse PD-L1 3-'UTR through microRNA.org and found that mouse PD-L1 was also potentially targeted by mouse miR-195 and miR-16 (Fig. 5A). To confirm this targeting, we generated reporter constructs containing the potential binding site of the PD-L1 gene 3'-UTR regions or their mutants. The luciferase activity of PD-L1 wildtype constructs was inhibited by miR-195 and miR-16 overexpression (Fig. 5B) in Trampc1 cells. Point mutations in the putative binding site abrogated the inhibition by miR-195 and miR-16 (Fig. 5B), which demonstrated that miR-195 and miR-16 specifically targeted the PD-L1 3'-UTR. Western blotting analyses indicated that miR-195 and miR-16 overexpression in Trampc1 cells decreased the protein levels of PD-L1 (Fig. 5C). Furthermore, FCM analysis showed cell surface PD-L1 expression was significantly higher in radiation treated Trampc1 cells (24-72h after 20Gy radiation) compared with untreated cells (Fig. 5D). Restoration of miR-195 and miR-16 expression in Trampc1 cells resulted in decreased cell-surface PD-L1 expression (Fig. 5D).

To investigate the role of miR-195 and miR-16 in regulating Trampc1 tumor growth in vivo, we transplanted Trampc1 cells stably overexpressing miR-195 or miR-16 into syngeneic Tramp mice, followed by anti-PD-L1 treatment. The mouse tumor volumes are shown in Fig

Fig. 5. PD-L1 is required for the efficacy of miR-195 and miR-16 treatment in Trampc1 tumors. (A) The miR-195 and miR-16 targeting sites in 3'-UTR of mouse PDL1 were shown. (B) The reporter constructs containing the mouse wild-type (WT) and mutant (MUT) PD-L1. 3'- UTR regions were cotransfected into Trampc1 cells with miR-195 or miR-16. The relative Luciferase/Renila activities were analyzed in the cells $48 \mathrm{~h}$ after the transfection. Results represent the mean \pm SEM from three independent experiments. ${ }^{* *} \mathrm{p} \leq$ 0.01 . (C) Trampc1 cells were transfected with miR-195 or miR-16. miR-195 and miR-16 mRNA levels were determined by qRT-PCR assay. Results represent the mean \pm SEM from three independent experiments. ${ }^{* *} \mathrm{p} \leq 0.01$. Expression levels of PD-L1 were analyzed by western blotting. One representative experiment out of three is shown. (D) Trampc1 cells treated with mir-195/16 mimics or radiation (20Gy) for 24-72h. The expression of cell surface PD-L1 was

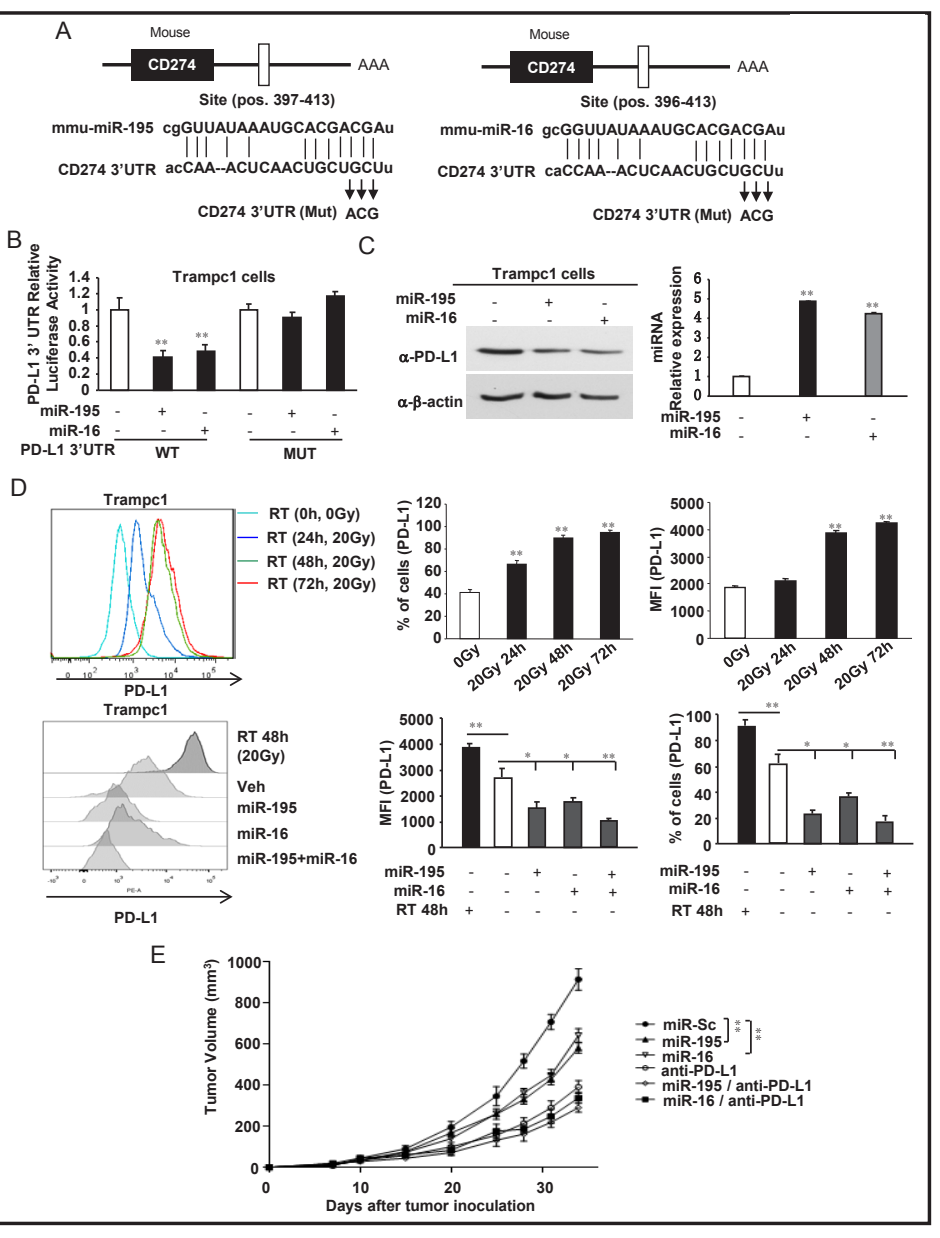
determined by FCM in Trampc1 cells. The results represent the mean \pm SEM from three independent experiments. ${ }^{*} \mathrm{p} \leq 0.05,{ }^{* *} \mathrm{p} \leq 0.01$. (E) Trampc1 cells $\left(5 \times 10^{6}\right)$ with stable overexpression of miR-195 or miR-16 were injected into syngeneic Tramp mice followed by anti-PD-L1 treatment. The mouse tumor volumes of the mice was characterized in different treatment groups. Bar graphs are shown as the mean \pm SEM ( $n=12$ mice/group). ** $\mathrm{p} \leq 0.01$. 
5E. miR-195 and miR-16 overexpression significantly decreased tumor volumes compared with miR-Sc in untreated Tramp mice, whereas anti-PD-L1 treatment eliminated the efficacy of miR-195 and miR-16 overexpression, suggesting that PD-L1 is required for miR-195 or miR-16 in Trampc1 tumors.

miR-195/-16 enhances the radiotherapy efficacy of Trampc1 tumors by regulating immunocyte production in the tumor microenvironment

To test the effect of combination treatment with miR-195/-16 and radiation in mouse prostate cancer, we implanted Trampc1 cells expressing miR-195/-16 into syngeneic Tramp mice, followed by radiation or anti-PD-L1treatment. Tumor volumes are shown in Fig. 6A. Radiation treatment significantly decreased the tumor volumes in Tramp mice with miR-195 and miR-16 overexpressing tumors, indicating that miR-195 and miR-16 might enhance the efficacy of radiation treatment in suppressing the progression of Trampc1 tumors (Fig 6A). miR-195 and miR-16 overexpression alone exhibited inhibitory effects on tumor growth, which indicated that miR-195 and miR-16 might function as a tumor suppressor (Fig. 6A). To address whether combination therapy resulted in the generation of prolonged protective $\mathrm{T}$ cell immunity, 30 days after complete tumor rejection, Tramp mice were re-challenged with a much higher dose of Trampc1 tumor cells on the opposite flank. No palpable tumors were detected in the treated mice after a few weeks, whereas tumors were palpable after 1 week in naive mice (Fig. 6B).

Regulatory T cells (Tregs) and Myeloid-derived suppressor cells (MDSCs) are believed to be the major components of the immune suppressive cells in the tumor microenvironment

Fig. 6. miR-195/-16 enhanced the radiotherapy efficacy of Trampc1 tumors by regulating immunocyte production in the tumor microenvironment. Trampc1 cells $\left(5 \times 10^{6}\right)$ with stable overexpression of miR-195/-16 were implanted into syngeneic Tramp mice followed by radiation or anti-PD-L1 treatment. (A) Tumor volumes of the mice were characterized in different treatment groups. (B) Tumor-free mice that underwent combination therapy were resistant to the tumor re-challenge. Thirty days after tumor eradication the mice were re-challenge with Trampc1 cells on the opposite flank. (C) CD8+ T cells, Tregs, and MDSCs were isolated from the tumors of Trampc1 tumor-bearing mice and counted by FCM analysis. CD8+ TIL (CD45+) infiltration as a percentage of total leucocytes. Changes in CD4+ CD25+ Foxp3+ Tregs as a percentage of TILs. Representative of MDSCs gated on a CD45+ cell populations are shown. Bar graphs are shown as the mean \pm SEM ( $\mathrm{n}=$ 12 mice/group). * $\mathrm{p} \leq 0.05$, ** $\mathrm{p} \leq$ 0.01 .

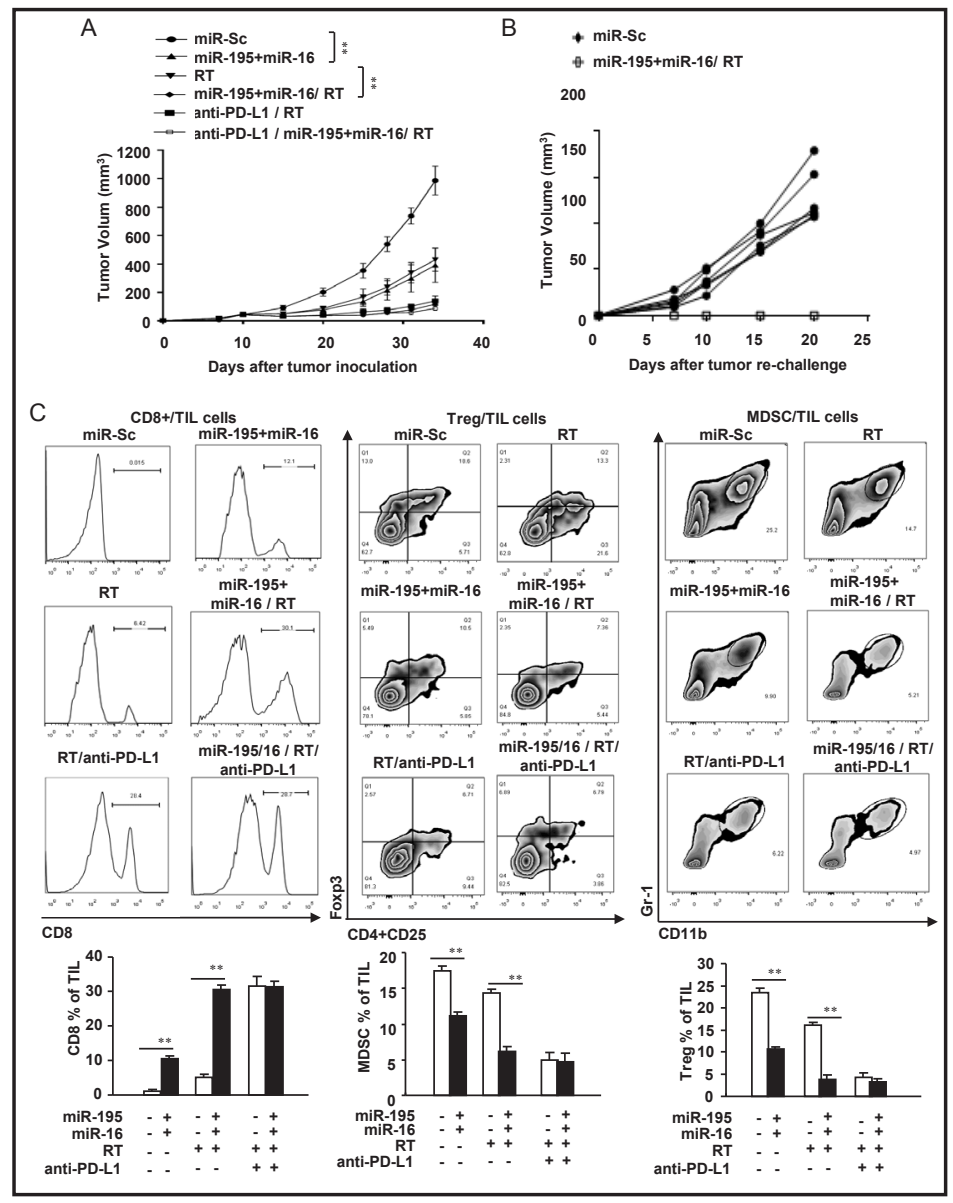


and have been associated with radioresistance [22]. These two cell types have been shown to be expanded in preclinical tumor models and to promote $\mathrm{T}$ cell dysfunction that favors tumor progression [22]. Our present work demonstrated that PD-L1 is required for miR195 and miR-16 to inhibit Trampc1 tumor growth. We examined the abundance of CD8+ T cells, MDSCs and Tregs in Trampc1 tumors using a FACScan flow cytometer and the same animal model. As shown in Fig. 6C, radiation treatment significantly increased the number of CD8+ T cells and decreasedMDSC and Treg levels in the Tramp mice with miR-195 and miR-16 overexpressing tumors, whereas anti-PD-L1 treatment blocked the efficacy of the combination treatment. These results indicate that miR-195 and miR-16 might activate the T cell immune response and repress $T$ cell dysfunction during radiotherapy through a PD-L1dependent pathway. Taken together, these findings further demonstrated that miR-195 and miR-16 enhanced the efficiency of radiotherapy by regulating CD8+ T cell, MDSC and Treg abundance in the tumor microenvironment through a PD-L1-dependent pathway.

miR-195/-16 enhances the radiotherapy efficacy of Trampc1 tumors byactivating cytotoxic $T$ cells and reducing regulatory cytokine secretions in the tumor microenvironment

Antigen-specific cytotoxic T cells (CTLs) play indispensable role in the immune response to infectious pathogens [23]. IFN- $\gamma$ expression is a surrogate marker of CTL levels [23]. As shown in Fig. 7A, radiation therapy significantly increased the number of IFN- $\gamma+/$ CD8+ T cells in Tramp mice with miR-195 and miR-16 overexpressing tumors, whereas anti-PD-L1 treatment eliminated the efficacy of the combination treatment, which suggested that miR195 and miR-16 also enhanced the efficacy of radiotherapy by CTL activation through a PDL1-dependent pathway.

We have shown that miR-195/-16 directly regulates PD-L1, and also that miR-195/-16 blocks the expression of PD-L1 to regulate T cell cytokine secretions in a DU145/T cell coculture model. Subsequently, we measured the cell surface PD-L1 expression in Trampc1 tumor cells. FCM analysis showed that cell surface PD-L1 expression was significantly increased following treatment with radiation alone (Fig. 7B). Restoration of miR-195 and miR16 expression in radiation-treated Trampc1 tumors resulted in decreased cell surface PD-L1 expression (Fig. 7B). Furthermore, we measured the mRNA levels of PD-L1, CD80, CD8, TNF- $\alpha$ and IFN- $\gamma$ via qRT-PCR in Trampc1 tumors. A similar result was observed. As shown in Fig. 7C, the mRNA levels of PD-L1 and CD80 significantly increased in Trampc1 tumors following radiation therapy alone. Restoration of miR-195 and miR-16 expression in radiation-treated Trampc1 tumors resulted in decreased PD-L1 and CD80 expression. Furthermore, the mRNA levels of CD8, TNF- $\alpha$ and IFN- $\gamma$ were increased in Trampc1 tumors following treatment with radiation alone. The effects of radiation on $\mathrm{CD} 8$, TNF- $\alpha$ and IFN- $\gamma$ were enhanced when miR195 and miR-16 were overexpressed (Fig. 7C). We subsequently measured T cell cytokine secretions in the serum of Tramp mice. As shown in Fig. 7D, radiation treatment did not inhibit the secretion of IL-10 and TGF- $\beta$ in the Tramp mice carrying miR-Sc-overexpressing tumorsbut clearly inhibited the secretion of IL-10 and TGF- $\beta$ in the Tramp mice with miR-195 and miR-16 overexpressing tumors. Moreover, radiation treatment increased the secretion of TNF- $\alpha$ and IFN- $\gamma$ in the Tramp mice carrying miR-Sc overexpressing tumors. However, radiation treatment significantly induced the secretion of TNF- $\alpha$ and IFN- $\gamma$ in the Tramp mice with miR-195 and miR-16 overexpressing tumors (Fig. 7D), which suggested that miR195/-16 enhanced the efficacy of the radiotherapy by regulating the secretions of regulatory cytokines. Taken together, these findings demonstrated that miR-195/-16 might improve the immune evasion of radiotherapy-induced PD-L1-associated T cells through PD-L1 signaling (Fig. 7E).

\section{Discussion}

Unfortunately, many more patients will experience increasing levels of prostate-specific antigen (PSA) following radiotherapy, a condition BCR [24]. Physicians treating patients with BCR are faced with a difficult series of decisions, attempting to delay the occurrence of 
Fig. 7. miR-195/-16 enhance the radiotherapy efficacy of Trampc1 tumors by reducing regulatory cytokine secretions in the tumor microenvironment. Trampc1 cells $\left(5 \times 10^{6}\right)$ with stable overexpression of miR-195/-16 were implanted into syngeneic Tramp mice, followed by radiation or anti-PD-L1 treatment. $\mathrm{T}$ cells were harvested from regressing tumors and stained with various markers. Mouse circulating serum was collected $72 \mathrm{~h}$ after radiation treatment. (A) IFN- $\gamma+/ \mathrm{CD} 8+$ $\mathrm{T}$ cells were isolated from tumors of Trampc1 tumorbearing mice and counted. $* \mathrm{p} \leq 0.05,{ }^{* *} \mathrm{p} \leq 0.01$. Bar graphs are shown as mean $\pm \operatorname{SEM}(\mathrm{n}=12$ mice/ group). (B) Expression of cell surface PD-L1 was determined by FCM in Trampc1 tumors. * $\mathrm{p} \leq 0.05$, ** $\mathrm{p} \leq 0.01$. Bar graphs are shown as the mean \pm SEM ( $\mathrm{n}=12 \mathrm{mice} /$ group) (C) Relative expression levels of

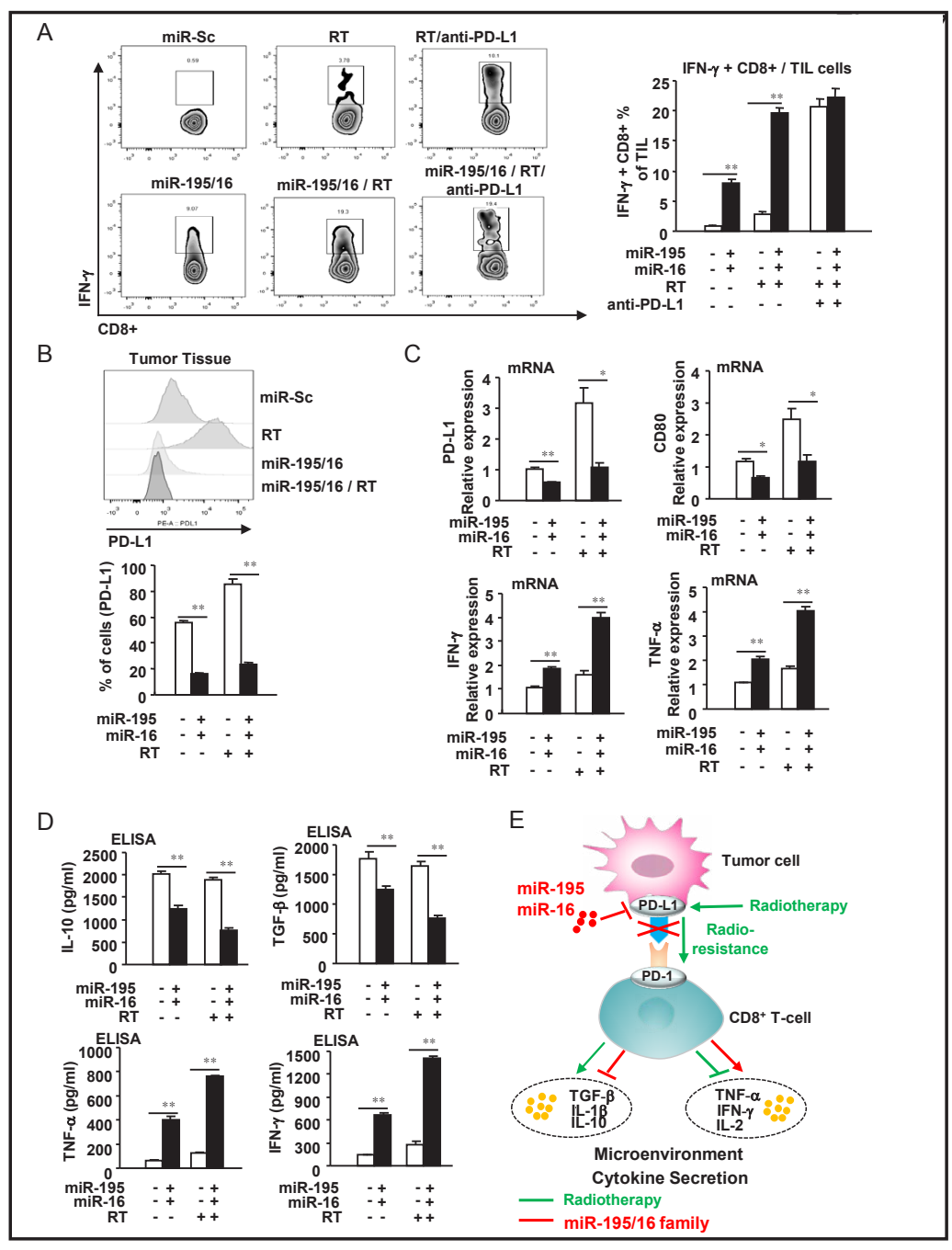
PD-1, PD-L1, CD80, TNF- $\alpha$ and IFN- $\gamma$ from tumors of Trampc1 tumor-bearing mice were determined via qRT-PCR assay. ${ }^{*} p \leq 0.05,{ }^{* *} p$ $\leq 0.01$. Bar graphs are shown as mean \pm SEM $(n=12$ mice/group). (D) Circulating serum from Tramp mice was assayed for IL-10, TNF- $\alpha$, IFN- $\gamma$, and TGF- $\beta$ by cytokine ELISA. ** $p \leq 0.01$. Bar graphs are shown as the mean \pm SEM ( $n=12$ mice/group). (E) Schematic representation of the biological and functional interaction between PD-L1 and radiotherapy through the miR-195/-16 family regulatory cascade.

metastatic disease and death while keeping patients whose disease may never affect their overall survival or quality of life from being over-treated [24]. Radiotherapy is a standard treatment option for both organ-confined and regionally advanced prostate cancer [25]. Radiotherapy enhances tumor immunogenicity and antigen presentation, increases cytokine production, and alters the microenvironment of tumor cells. Radiation-induced adaptive immunity has the potential to improve outcomes in aggressive prostate cancer because it involves high levels of PD-L1 expression [26]. Additionally, fractionated radiotherapy induces the up-regulation of PD-L1 through CD8+ T cell production of IFN- $\gamma$. Therefore, further investigation into the mechanisms regulating the immune response have revived interest into the use of combination radiation and immune-based therapies to improve local control and prolong BCR-free survival in prostate cancer. The evidence shows that a growing number of miRNAs have been identified to be important regulators of radiosensitivity in kinds of tumors [27-29], however, the mechanism remains unclear.Here, we found that high expression levels of miR-195 and miR-16 were positively correlated with the BCR-free

\section{KARGER}


survival time of prostate cancer patients but inversely correlated with PD-L1, PD-1, CD80 and CTLA-4 expression in this dataset. Functional investigations revealed that miR-195/16 induced an immune response to radiotherapy via activation of the $\mathrm{T}$ cell response by blocking the PD-L1 immune checkpoint. These results indicated that endogenous miR-195/16 plays an important role in the radioresistance of prostate cancer. Suppression of PD-L1 may be possible by promoting the expression of endogenous miR-195 and miR-16, thereby improving the therapeutic index of the radioresistance-based increase in PD-L1 expression.

A MRX34 clinical trial is currently underway at MD Anderson. MRX34, an investigational drug with the ability to mimic the tumor-suppressing capacity of miR-34, increases CD8+ $\mathrm{T}$ cells of the immune system when combined with radiotherapy [30]. Gene therapy based on miRNA provides an attractive antitumor approach to comprehensive cancer therapy. In our previous work, we found that miR-424(322) could reverse chemoresistance via T-cell activation by blocking PD-L1 expression in ovarian cancer [17]. In the current study, mechanistic investigations indicated that the miR-195 and miR-16 family also induced an immune response by blocking the PD-L1 immune checkpoint of radiotherapy in prostate cancer. Our research revealed a novel mechanism by which this miRNA family influenced the PD-L1/PD-1 signaling pathway. Further studies are needed to confirm these findings in other tumors.

By creating a tumor immune environment that allows the lesion to lude immune system attack, cancer cells that grow to form invasive tumors may escape the immune system [31]. Modification of the tumor microenvironment can involve the down regulation of major histocompatibility complex molecules or the induction of immune inhibitory cytokines and chemokines, which leads directly to activated T cells and the proliferation and activation of immunosuppressive cell populations such as Tregs and MDSCs [32]. In this study, the tumor immune environment could be actively manipulated by the miR-195/-16 family to revert the tumor environment, allowing the activation of CD8+ T cells, inhibition of MDSCs and Tregs, and induction of cytokines such as IFN- $\gamma$, TNF- $\alpha$, and IL-2 that are associated with cancer eradication.

A recent study demonstrated that blockage of PD-L1 restored immune suppression and resulted in the expansion of tumor-infiltrating lymphocytes (TILs) [33]. An increased number of CD4 and CD8+ cells is related to higher CD8/Treg and CD4/Treg ratios [34]. This is related to a moderate clinical response, together with molecular evidence of functional activation of TILs. A parallel increase in MDSCs reveals the archetypal tumor immunosuppressive environment [35]. In this model, miR-195/-16 in combination with radiotherapy was shown to be highly effective. This effect is likely due to the downregulation of high expression levels of PD-L1 by miR-195/-16 in Trampc1 tumor cells as well as tumor-derived myeloid cells. The current research results show that tumors lacking TILs may have PD-L1 mediated suppression, leading to TIL depletion. Our findings suggest that high frequency of Tregs and MDSCs in tumors lacking TILs is associated with poorly performing Tregs in human prostate cancer. Our work has shown, using the combination of miR-195/-16 and radiotherapy that miR-195/16 regulates the production of CD8+ T cells, MDSCs and Tregs, which activate CTLs and reduce the secretions of regulatory cytokines and ultimately, enhance radiotherapy efficacy.

\section{Conclusion}

In summary, we identified a novel mechanism by which miR-195 and miR-16 influence PD-L1/PD-1 immune checkpoint signaling pathway. Restoration of the expression of miR195/-16 could enhance radiotherapy via activation of the T cell response in the tumor microenvironment by blocking the PD-L1 immune checkpoint. This synergy between radiotherapy and immunotherapy was accompanied by the proliferation of functional cytotoxic CD8+ T cells and inhibition of MDSCs and Tregs. This work indicates a biological and functional interaction between immunotherapy and radiotherapy through the miR195/-16 family regulatory cascade.

\section{KARGER}




\section{Cellular Physiology Cell Physiol Biochem 2018;48:801-814 and Biochemistry Published online: \begin{tabular}{l|l} 
DOI: 10.1159/000491909 20, 2018 & $\begin{array}{l}\text { (c) } 2018 \text { The Author(s). Published by S. Karger AG, Basel } \\
\text { www.karger.com/cpb }\end{array}$
\end{tabular}}

Tao et al.: Mir-195/-16 Family Modulated Immune Response

\section{Acknowledgements}

This work was supported by grant from National Natural Sciences Foundation of China (81672524, 81602678, 81502227 and 81772762), Hubei Provincial Natural Science Foundation of China (2018CFA038), Independent Innovation Foundation of Huazhong University of Science and Technology (118530309) and Natural Science Foundation of Tianjin (17JCQNJC12300).

\section{Disclosure Statement}

The authors declare to have no competing interests.

\section{References}

1 Alberti C: Prostate cancer: radioresistance molecular target-related markers and foreseeable modalities of radiosensitization. Eur Rev Med Pharmacol Sci 2014;18:2275-2282.

2 Gubin MM, Zhang X, Schuster H, Caron E, Ward JP, Noguchi T, Ivanova Y, Hundal J, Arthur CD, Krebber WJ, Mulder GE, Toebes M, Vesely MD, Lam SS, Korman AJ, Allison JP, Freeman GJ, Sharpe AH, Pearce EL, Schumacher TN, Aebersold R, Rammensee HG, Melief CJ, Mardis ER, Gillanders WE, Artyomov MN, Schreiber RD: Checkpoint blockade cancer immunotherapy targets tumour-specific mutant antigens. Nature 2014; 515:577-581.

3 Quezada SA, Peggs KS, Simpson TR, Allison JP: Shifting the equilibrium in cancer immunoediting: from tumor tolerance to eradication. Immunol Rev 2011;241:104-118.

4 Pardoll DM. The blockade of immune checkpoints in cancer immunotherapy. Nat Rev Cancer 2012;12:252264.

5 Wainwright DA, Chang AL, Dey M, Balyasnikova IV, Kim CK, Tobias A, Cheng Y, Kim JW, Qiao J, Zhang L, Han Y, Lesniak MS: Durable therapeutic efficacy utilizing combinatorial blockade against IDO, CTLA-4, and PDL1 in mice with brain tumors. Clin Cancer Res 2015;21:662.

-6 Xu C, Fillmore CM, Koyama S, Wu H, Zhao Y, Chen Z: Loss of Lkb1 and Pten leads to lung squamous cell carcinoma with elevated PD-L1 expression. Cancer Cell 2014;25:590-604.

7 Ngiow SF, McArthur GA, Smyth MJ: Radiotherapy complements immune checkpoint blockade. Cancer Cell 2015;27:437-438.

8 Wu D, Li Y, Zhang H, Hu X: Knockdown of Lncrna PVT1 Enhances Radiosensitivity in Non-Small Cell Lung Cancer by Sponging Mir-195. Cell Physiol Biochem 2017;42:2453-2466.

-9 Lan F, Yue X, Ren G, Li H, Ping L, Wang Y, Xia T: miR-15a/16 enhances radiation sensitivity of non-small cell lung cancer cells by targeting the TLR1/NF-kappaB signaling pathway. Int J Radiat Oncol Biol Phys 2015;91:73-81.

10 Cai C, Chen QB, Han ZD, Zhang YQ He HC, Chen JH: miR-195 Inhibits Tumor Progression by Targeting RPS6KB1 in Human Prostate Cancer. Clin Cancer Res 2015;21:4922-4934.

-11 Bonci D, Coppola V, Musumeci M, Addario A, Giuffrida R, Memeo L, D’Urso L, Pagliuca A, Biffoni M, Labbaye C, Bartucci M, Muto G, Peschle C, De Maria R: The miR-15a-miR-16-1 cluster controls prostate cancer by targeting multiple oncogenic activities. Nat Med 2008;14:1271-1277.

-12 Wang T, Song W, Chen Y, Chen R, Liu Z, Wu L, Li M, Yang J, Wang L, Liu J, Ye Z, Wang C, Chen K: Flightless I Homolog Represses Prostate Cancer Progression through Targeting Androgen Receptor Signaling. Clin Cancer Res 2016;22:1531-1544.

-13 Taylor BS, Schultz N, Hieronymus H, Gopalan A, Xiao Y, Carver BS, Arora VK, Kaushik P, Cerami E, Reva B, Antipin Y, Mitsiades N, Landers T, Dolgalev I, Major JE, Wilson M, Socci ND, Lash AE, Heguy A, Eastham JA, Scher HI, Reuter VE, Scardino PT, Sander C, Sawyers CL, Gerald WL: Integrative genomic profiling of human prostate cancer. Cancer Cell 2010;18:11-22.

14 Wang T, Liu Z, Guo S, Wu L, Li M, Yang J, Chen R, Xu H, Cai S, Chen H, Li W, Wang L, Hu Z, Zhuang Q, Xu S, Wang L, Liu J, Ye Z, Ji JY, Wang C, Chen K: The tumor suppressive role of CAMK2N1 in castration-resistant prostate cancer. Oncotarget 2014; 5:3611-3621.

15 Wang T, Guo S, Liu Z, Wu L, Li M, Yang J, Chen R, Liu X, Xu H, Cai S, Chen H, Li W, Xu S, Wang L, Hu Z, Zhuang Q, Wang L, Wu K, Liu J, Ye Z, Ji JY, Wang C, Chen K: CAMK2N1 inhibits prostate cancer progression through androgen receptor-dependent signaling. Oncotarget 2014;5:10293-10306. 


\section{Cellular Physiology Cell Physiol Biochem 2018;48:801-814 \begin{tabular}{l|l} 
and Biochemistry & $\begin{array}{l}\text { DOI: 10.1159/000491909 } \\
\text { Published on } 2018 \text { The Author(s). Published by S. Karger AG, Basel } \\
\text { www.karger.com/cpb }\end{array}$
\end{tabular}}

Tao et al.: Mir-195/-16 Family Modulated Immune Response

16 Schnurr M, Galambos P, Scholz C, Then F, Dauer M, Endres S, Eigler A: Tumor cell lysate-pulsed human dendritic cells induce a T-cell response against pancreatic carcinoma cells: an in vitro model for the assessment of tumor vaccines. Cancer Res 2001;61:6445-6450.

17 Xu S, Tao Z, Hai B, Liang H, Shi Y, Wang T, Song W, Chen Y, OuYang J, Chen J, Kong F, Dong Y, Jiang SW, Li W, Wang P, Yuan Z, Wan X, Wang C, Li W, Zhang X, Chen K: miR-424(322) reverses chemoresistance via T-cell immune response activation by blocking the PD-L1 immune checkpoint. Nat Commun 2016;7:11406.

18 Ha TY: The Role of MicroRNAs in Regulatory T Cells and in the Immune Response. Immune Netw 2011;11:11-41.

19 Hoeppli RE, Wu D, Cook L, Levings MK: The environment of regulatory T cell biology: cytokines, metabolites, and the microbiome. Front Immunol 2015;6:61.

20 Carbotti G, Nikpoor AR, Vacca P, Gangemi R, Giordano C, Campelli F, Ferrini S, Fabbi M: IL-27 mediates HLA class I up-regulation, which can be inhibited by the IL-6 pathway, in HLA-deficient Small Cell Lung Cancer cells. J Exp Clin Cancer Res 2017;36:140.

21 Blank C, Gajewski TF, Mackensen A: Interaction of PD-L1 on tumor cells with PD-1 on tumor-specific T cells as a mechanism of immune evasion: implications for tumor immunotherapy. Cancer Immunol Immunother 2005;54:307-314.

22 Lindau D, Gielen P, Kroesen M, Wesseling P, Adema GJ: The immunosuppressive tumour network: myeloidderived suppressor cells, regulatory T cells and natural killer T cells. Immunology 2013;138:105-115.

-23 Ghanekar SA, Nomura LE, Suni MA, Picker LJ, Maecker HT, Maino VC: Gamma interferon expression in CD8(+) T cells is a marker for circulating cytotoxic T lymphocytes that recognize an HLA A2-restricted epitope of human cytomegalovirus phosphoprotein pp65. Clin Diagn Lab Immunol 2001;8:628-631.

24 Paller CJ, Antonarakis ES: Management of biochemically recurrent prostate cancer after local therapy: evolving standards of care and new directions. Clin Adv Hematol Oncol 2013;11:14-23.

-25 Rose BS, Chen MH, Wu J, Braccioforte MH, Moran BJ, Doseretz DE, Katin MJ, Ross RH, Salenius SA, D’Amico AV: Androgen Deprivation Therapy Use in the Setting of High-dose Radiation Therapy and the Risk of Prostate Cancer-Specific Mortality Stratified by the Extent of Competing Mortality. Int J Radiat Oncol Biol Phys 2016;96:778-784.

26 Gevensleben H, Dietrich D, Golletz C, Steiner S, Jung M, Thiesler T, Majores M, Stein J, Uhl B, Müller S, Ellinger J, Stephan C, Jung K, Brossart P, Kristiansen G: The Immune Checkpoint Regulator PD-L1 Is Highly Expressed in Aggressive Primary Prostate Cancer. Clin Cancer Res 2016;22:1969-1977.

-27 Song Y, Zuo Y, Qian XL, Chen ZP, Wang SK, Song L, Peng LP: Inhibition of MicroRNA-21-5p Promotes the Radiation Sensitivity of Non-Small Cell Lung Cancer Through HMSH2. Cell Physiol Biochem 2017;43:12581272.

28 Jiang LP, Zhu ZT, Zhang Y, He CY: Downregulation of MicroRNA-330 Correlates with the Radiation Sensitivity and Prognosis of Patients with Brain Metastasis from Lung Cancer. Cell Physiol Biochem 2017;42:2220-2229.

29 Wang Y, Wu J, Guo W, Sun Q, Chen X, Zang W, Dong Z, Zhao G: $\alpha$-Solanine Modulates the Radiosensitivity of Esophageal Cancer Cells by Inducing MicroRNA 138 Expression. Cell Physiol Biochem 2016;39:996-1010.

-30 Cortez MA, Ivan C, Valdecanas D, Wang X, Peltier HJ, Ye Y, Araujo L, Carbone DP, Shilo K, Giri DK, Kelnar K, Martin D, Komaki R, Gomez DR, Krishnan S, Calin GA, Bader AG, Welsh JW: PDL1 Regulation by p53 via miR-34. J Natl Cancer Inst 2015;108. pii: djv303.

-31 Gajewski TF, Schreiber H, Fu YX: Innate and adaptive immune cells in the tumor microenvironment. Nat Immunol 2013;14:1014-1022.

32 Mauge L, Terme M, Tartour E, Helley D: Control of the adaptive immune response by tumor vasculature. Front Oncol 2014;4:61.

-33 Duraiswamy J, Freeman GJ, Coukos G: Therapeutic PD-1 pathway blockade augments with other modalities of immunotherapy T-cell function to prevent immune decline in ovarian cancer. Cancer Res 2013;73:690012.

34 Alvarez Arias DA, Kim HJ, Zhou P, Holderried TA, Wang X, Dranoff G, Cantor H. Disruption of CD8+ Treg activity results in expansion of $\mathrm{T}$ follicular helper cells and enhanced antitumor immunity. Cancer Immunol Res 2014;2:207-216.

-35 Abad C, Nobuta H, Li J, Kasai A, Yong WH, Waschek JA: Targeted STAT3 disruption in myeloid cells alters immunosuppressor cell abundance in a murine model of spontaneous medulloblastoma. J Leukoc Biol 2014;95:357-67. 